\title{
Research on Problems and Countermeasures of Information Technology in Classroom Teaching Application
}

\author{
Liu Xudong ${ }^{1}$, , Hao $\mathrm{Qi}^{2}$, Zhang Liguo ${ }^{1}$ \\ ${ }^{1}$ Faculty of Information Technology, Beijing University of Technology, Beijing, China \\ ${ }^{2}$ College of Teacher Education, Shandong Normal University, Jinan, China
}

\section{Email address:}

hicx@bjut.edu.cn (Liu Xudong)

${ }^{*}$ Corresponding author

\section{To cite this article:}

Liu Xudong, Hao Qi, Zhang Liguo. Research on Problems and Countermeasures of Information Technology in Classroom Teaching Application. Science Discovery. Vol. 6, No. 5, 2018, pp. 373-379. doi: 10.11648/j.sd.20180605.20

Received: September 20, 2018; Accepted: October 15, 2018; Published: October 18, 2018

\begin{abstract}
At present, countries all over the world are vigorously promoting the development of education informatization. The core of education information is the integration of information technology and curriculum, and the application of information technology classroom teaching is the concrete realization of this thought in teaching. This paper focuses on the problems and countermeasures of information technology in classroom teaching applications. Firstly, it briefly reviews the development process of education informatization in China, and summarizes the main contents of classroom teaching reform under the background of informatization and the role of information technology in promoting classroom teaching reform. Then it discusses in detail the 4 specific problems of information technology applied in classroom teaching, that is, the positioning of information technology in teaching is not clear, the awareness and ability of teachers' application technology are lacking, the application of information technology in the classroom is inappropriate, and the interactive feedback in the information environment is poor. Finally, the countermeasures are given, that is, the application of information technology in classroom teaching should focus on the organic integration of information technology and classroom teaching, enhance teachers' technical application ability, expand communication channels and achieve diversified evaluation, so as to achieve the purpose of optimizing classroom teaching, improving teaching quality, and facilitating the cultivation of talents in the information age.
\end{abstract}

Keywords: Education Informatization, Information Technolgy, Classroom Teaching, Countermeasures

\section{信息技术在课堂教学应用中的问题与对策研究}

刘旭东 ${ }^{*}$, 郝琪 $^{2}$, 张利国 $^{1}$

1信息学部, 北京工业大学, 北京, 中国

2教师教育学院, 山东师范大学, 济南, 中国

\section{邮箱}

hicx@bjut.edu.cn（刘旭东）

摘要：当前世界各国都在大力推动教育信息化的发展。教育信息化的核心是信息技术与课程整合，而信息技术的课堂 教学应用正是这一思想在教学中的具体实现。本文针对信息技术在课堂教学应用中的问题和对策展开研究。首先简要 回顾了中国教育信息化发展历程，并对信息化背景下课堂教学变革的主要内容及信息技术促进课堂教学变革的作用点 进行了文献综述; 然后详细论述了信息技术应用于课堂教学中所存在 4 点具体问题,即信息技术在教学中的定位不明确、 教师应用技术的意识与能力有欠缺、信息技术在课堂上的应用不恰当以及信息化环境下的互动反馈贫乏; 最后给出了 
应对策略, 即信息技术在课堂教学中的应用应该着眼于信息技术与课堂教学的有机融合, 提升教师的技术应用能力, 拓展交流渠道并实现多元化评价, 从而达到优化课堂教学、提高教学质量、更利于信息时代人才的培养的目的。

关键词：教育信息化，信息技术，课堂教学，应对策略

\section{1. 引言}

近年来随着计算机技术、通讯技术和互联网技术为代 表的信息技术的飞速发展，人类社会已经进入高度信息化， 知识正以前所未有的速度不断更新。教育与现代信息技术 的融合不仅会使人类的教育方式和学习方式发生重大变 化, 而且会对我们的教育思想、教学观念、教学模式、教 学内容和管理方法产生深刻影响[1]。

教育信息化是指在教育领域全面深入地运用现代信 息技术来促进教育改革与发展的过程。目前, 世界各国教 育部门都在大力推动信息技术在教育领域的应用。2005 年，英国教育部门发布了“利用技术促进学习计划”; 2008 年，澳大利亚教育部门发布了“数字教育改革计划”; 2010 年，美国教育部门发布了主题为“变革美国教育：以技术 赋能学习”的国家教育技术规划 (National Education Technology Program, 简称NETP)。这些规划都高度认同 信息技术对教育产生的巨大的变革, 高度肯定了信息技术 在当前乃至未来教育中所起的重要作用, 并且寄希望于信 息技术未来能进一步推动教育系统的整体性变革。

中国也同样高度关注教育信息化在提高国民素质和 创新能力方面所起的重要作用。2001年教育部颁布的《基 础教育课程改革纲要（试行）》中在第 11 条明确规定要大 力推进信息技术在教学过程中的普遍应用, 促进信息技术 与学科课程的整合[2]; 2010年教育部在《国家中长期教育 改革和发展规划纲要（2010-2020年）》明确指出要“探索 现代信息技术与教育的全面深度融合, 以信息化引领教育 理念和教育模式的创新”、“信息技术对教育发展具有革命 性影响, 必须予以高度重视”[3]; 2012年教育部在《教育 信息化十年发展规划（2011-2020年）》中指出“高等教育 信息化是促进高等教育改革创新和提高质量的有效途径”, 要“注重信息技术与教育的全面深度融合”[4]。可见，国家 把教育信息化看作一种政府行为, 并随着时代的发展制订 出一系列相关政策及法规, 表现出对信息技术在教学中 应用的高度重视。

自新课程改革以来, 信息技术已被广泛应用于课堂教 学中, 大多教师逐渐体会到信息技术应用于课堂教学的优 越性, 开始积极运用信息技术组织课堂, 课堂教学已经由 原来单一的授受式教学向多样化教学转变, 教学结构正在 发生质变。正确把握和处理教学与技术的关系是保证教学 健康发展的基本前提[5]。目前, 信息技术指导下的教学改 革还不够成熟，信息技术与课堂教学的融合仍然存在问题。 本文首先介绍了中国教育信息化发展概况, 简要回顾了中 国教育信息化发展历程, 并对信息化背景下课堂教学变革 的主要内容及信息技术促进课堂教学变革的作用点进行 了文献综述; 然后详细论述了信息技术应用于课堂教学中 所存在 4 点具体问题: 在教学中的定位不明确、教师的意
识与能力有欠缺、课堂应用不恰当、互动反馈贫乏; 最后 给出了应对策略, 即信息技术在课堂教学中的应用应该着 眼于信息技术与课堂教学的有机融合, 提升教师的技术应 用能力, 拓展交流渠道, 实现多元化评价。

\section{2. 中国教育信息化发展概况}

\section{1. 中国教育信息化发展历程}

回顾中国教育信息化发展历程, 大致可以分为三个阶 段, 如图1。第一个阶段是上世纪八、九十年代的萌芽阶 段, 这一阶段信息技术刚刚起步, 互联网还没有得到大规 模普及应用, 因此教育信息化技术主要以建设多媒体视听 教室、远程教学系统等为主, 这一阶段末期开始建设中国 教育科研计算机网; 第二个阶段是本世纪初的第一个十年, 在新一轮基础教育课程改革的推动下, 要求课堂教学变革 的呼声日益高涨，此时信息技术已在教育领域得到广泛而 深入的应用, 进入了快速发展阶段。这一阶段由于互联网 技术的迅速发展, 网络信息资源呈现出一种爆炸式的增长 态势, 教育信息化主要内容是各类教育/学科资源、各种 网络教学支持平台以及国家精品课程、教育资源库的建设; 第三个阶段是2010年至今, 这一阶段随着互联网+、云计 算和大数据等概念的提出和技术的发展, 慕课 (MOOC)、 翻转课堂、数字校园、微课程等新型的教育教学形态不断 涌现，标志着教育信息化进入了深入推广阶段。

\section{2. 信息化背景下课堂教学变革的主要内容}

教学过程是一个由教师、学生、教学内容、教学媒体 四个要素构成的稳定结构。任何形式的变革都是围绕这四 要素展开的。因此信息化背景下课堂教学变革的主要内容 可以概括为教师教的变革、学生学的变革、教学内容的变 革以及教学模式的变革。

关于教师教的变革, 多数研究者都是从教师的教学角 色、教学方式、教学设计这几个方面进行论证。如余胜泉、 陈玲 (2007) 通过案例研究分析发现网络环境下教师已经 不在局限于传授者角色, 而是逐渐转变为学习活动的指导 者、组织者与参与者, 教师的教学设计也从关注教学内容 转变为关注教学过程[6]。王宇飞 (2007) 认为信息技术环 境丰富了教学信息, 拓宽了信息的传递与反馈渠道, 学生 变得更加主动, 师生关系更加和谐[7]。

关于学生学的变革, 研究主要集中在学生学习角色、 学习方式上。如祝智庭 (2014) 在结合技术演进及实践热 点的基础上提到“当前移动计算技术、泛在计算技术、移 动设备的发展为学生创造了更加丰富的学习情境, 学生借 助移动设备实现学习情景和学习方式的切换, 更好地融合 正式学习与非正式学习, 同时基于情境感知, 学习者可主 动获取知识”[8]。又如徐松 (2013) 提到交互式环境给予 
学生主动参与的可能性, 学生不再是被动的接收信息, 而 是主动的获取信息 [9]。

关于教学内容的变革, 大多都是围绕阅读方式、写作 方式进行阐述。如广锦耀（2001）在论述信息技术应用所 引发的课堂教学变革中总结出当前信息技术所导致的阅 读方式的变革表现为从文本阅读走向超文本阅读、从单纯 阅读文字资料发展到阅读多媒体的电子读物 [10]。

教学模式是教学过程四要素相互作用所构成的一个 稳定的框架。能否打破传统的教学模式, 是当前教学改 革的关键。在传统教学模式下, 教学内容封闭, 教学手 段单一, 教学质量不高, 不利于学生主体性和能动性的 培养, 不利于学生创造性思维和实践能力的培养。关于 教学模式的研究主要集中在两个方面, 一是研究围绕教 学过程四要素展开。如侧重探讨师生角色关系的, 有人 提出了基于多媒体网络教室的“双主”教学模式、基于多 媒体教室的协作教学模式、基于计算机反馈系统的交互 教学模式; 侧重学生学习活动的, 如从学习活动的性质 和组织形式钟志贤教授 (2005) 归纳出出信息化教学模 式的类型有“个体-接受、群体-接受、个体-探究、群体探究”四大基本类型 [11]。二是研究基于整合的理念。如 何克抗、吴娟（2008）从信息技术与教育实现深度融合 的过程这一切入点提出了必须实施能有效变革课堂教学 结构的“教学模式”, 提出了“传递一接受”模式、“探究性”
教学模式、“研究性学习”教学模式、“适时教学”模式, 并展示了实施步骤和实施过程[12]。

\section{3. 信息技术促进课堂教学变革的作用点}

信息技术促进课堂教学变革的作用点即信息化背景 下课堂教学变革的原因。许多学者从信息技术的自身特征 及优势以及当前社会背景等角度探究信息技术在课堂教 学变革中的作用。祝智庭 (2014) 将信息技术的宏观优势 迁移到课堂教学中来阐述信息技术对教育变革的支撑作 用。他指出，信息技术的广泛应用打破了人类活动的时空 结构、改变了信息活动的社会主体结构以及参与方式、丰 富了信息的表现形式、提供了行为主体的智能代理功能。 技术的宏观优势渗透到课堂教学中, 改变了师生的教育关 系, 改变了学生的认知方式, 丰富了学习资源, 极大地增 加了人们的学习机会 $[8]$ 。梁文金鋾 (2013) 在《大数据— 课堂教学将迎来真正的变革》中总结出大数据是可以实现 课堂教学变革的关键即让每个学生都能以适合自己的方 式与节奏进行学习, 让每个学生都能够在自己原有的基础 上发展前进[13]。郭玉莲 (2012) 从教育思想、教育观念、 教学目标、教学手段、教学评价角度阐述传统教学已不能 适应时代发展的需要[14]。广锦耀 (2001) 则是从学生的 学与教师的教两个宏观角度指出信息技术在课堂教学中 的应用的前景十分广阔, 是改革传统教育教学的一个关键 切入点 $[10]$ 。

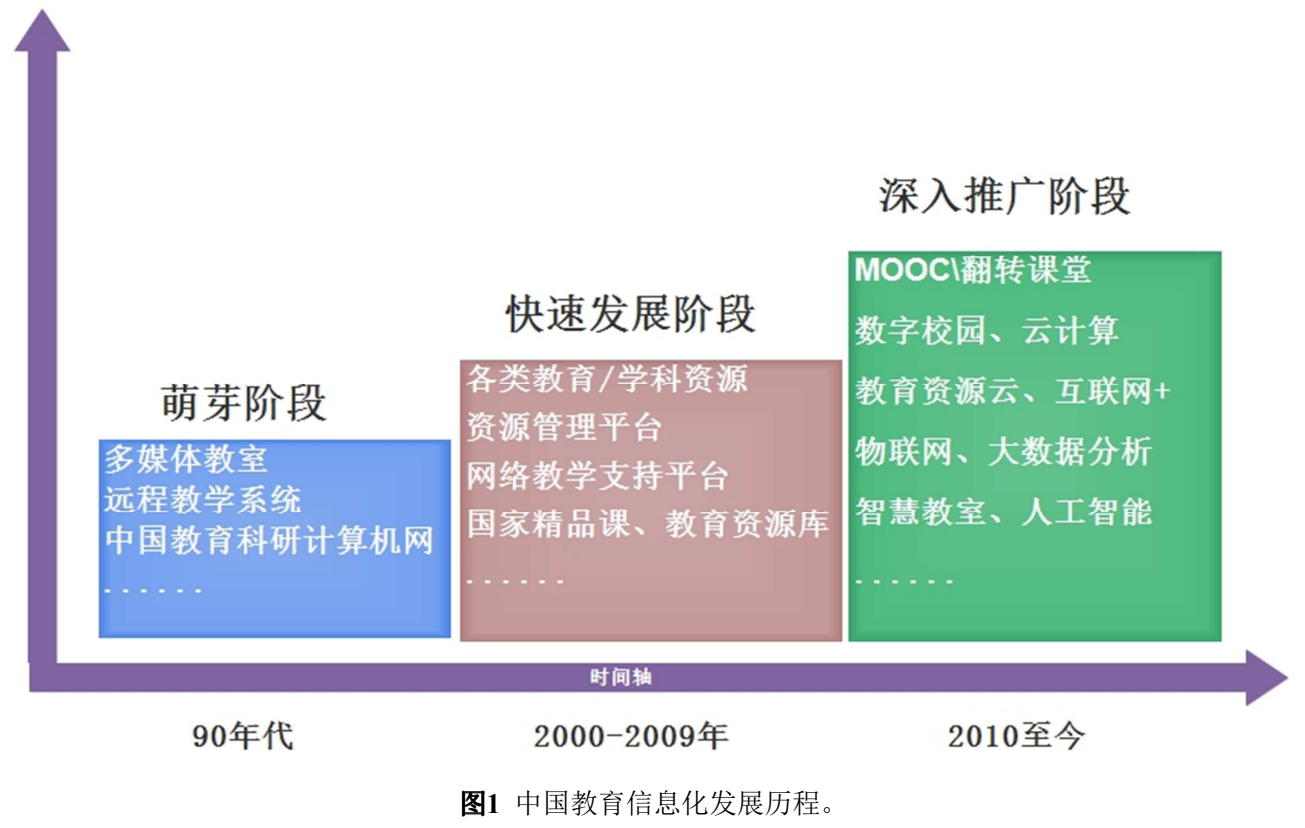

\section{3. 信息技术在课堂应用中出现的问题}

信息技术作为工具在课堂中表现出良好的辅助效果, 转变了传统的“粉笔+黑板”的教学方式和“书本十课堂”的 学习方式, 传统的教师主导的教学形式正在被师生合作和 小组合作的课堂所取代。然而信息技术的认知性作用的发 挥还存在很大的欠缺, 不能有效发挥在学生认知探究过程
的支持作用。可见信息技术变革的课堂教学还不够成熟, 还存在很多问题。

\section{1. 信息技术在教学中的定位不明确}

信息技术最初是作为辅助性手段应用于课堂教学的。 但随着技术发展以及现代化社会对人才要求的变化, 当前 社会对创新能力、批判性思维等高阶能力的要求越来越高, 从侧面反映出传统的授受式课堂已经不适应社会发展的 
需要, 因此应该明确信息技术在教学中的定位, 发挥信息 技术变革的作用, 促进课堂教学的发展。

当前教师在情境的创设、问题的导入以及组织教学等 方面发挥出积极的作用。例如教师利用信息技术作为教学 媒介为学生创设出形象生动的意境, 激发学生的兴趣, 带 动学生积极性和主动性。借助信息技术, 教师解决了传统 课堂需要显示图形图像的繁杂, 运用信息技术的媒体作用 通过课件生动地显示给学生, 转变了传统的教学方式, 提 高了课堂教学效率。可以看出, 信息技术在课堂教学中发 挥了很好的辅助作用, 信息技术作为辅助性手段便捷了教 师组织、处理教学问题。

但是现代化人才培养要求发挥学生的作用, 提高学生 主动探索的能力, 培养其创新、批判性思维的发展, 成为 一个全面发展的高素质人才。因此, 信息技术在教学中的 作用更多的是作为学生学习、交流和解决问题的工具。但 从案例中可以看出, 当前信息化课堂虽然意识到要发挥学 生的主体作用, 鼓励学生自主合作, 发展学生个性, 但是 具体到实践中, 信息技术环境的开放性、交互性、共享性、 大容量等优势没有得到淋漓尽致的发挥, 在运用技术支持 学生获取网络资源、支持学生在线互动与支持学生主动探 究等认知过程上的优势作用不显著。可见, 信息技术在课 堂教学中的应用仍处于辅助水平, 信息技术在教学中的定 位还不清晰。

\section{2. 教师应用技术的意识与能力显欠缺}

教师是信息技术手段的主要实施者, 教师运用技术的 意识与能力决定课堂教学的质量。因此, 教师应该首先具 备应有的技术素养, 保证课堂的进行。再之, 教师应该具 备应有的技术能力，保证技术优势的有效发挥。

信息技术环境的很多优势没有发挥出来，很大原因归 结于教师在课堂中的主导作用的缺失。例如, 在探究环节, 从形式上看, 教师做到组织学生进行自主学习或合作探究, 但是在技术利用上, 教师缺乏引导学生利用技术环境的互 动优势来组织沟通和协作的意识。例如, 在总结性评价中, 许多教师仍然局限于单一的评价, 缺乏用技术手段鼓励学 生进行展示和互评的意识, 有些教师甚至忽视了这一环节, 认为这是一个可有可无的部分。可见, 教师在技术环境下 的应用意识还很薄弱。

新型技术的应用并没有使技术的优势得到充分发挥， 这其中很大部分原因是由于教师在课堂中不能合理的使 用技术支持学生学习, 对信息化教学技术的应用停留在 “知其然不知其所以然”的境地, 将课堂教学的关注重点放 在课堂教学的环节和阶段上, 不能理解课堂教学改革的意 义和方向[15]。具体表现为教师技术应用的能力有限, 对 技术功能的认识有限, 对技术与课堂的融合理解有限, 使 得信息化教学模式的发挥受到限制, 不利于学生自主性的 发挥, 不利于学生个性的发展。

\section{3. 信息技术在课堂上的应用不恰当}

信息技术的有效应用是变革课堂的关键。信息技术与 课堂教学的有效融合, 能够转变教师与学生之间教与学的 方式, 变革传统的教学模式, 从而达到新课改要求, 适应
时代发展的需要。然而, 在现实教学中, 虽然技术为教学 选择和行动创造了新的可能性, 但也使得对这些可能性的 处置经常陷入一种不确定的状态 [16]。

教师应用信息技术手段的过程中呈现出一种“为了用 而用”的状态。例如在创设情境环节, 很多教师会综合使 用媒体、音像等为学生创设教学情境, 但是相当一部分教 师没有理解创设情境的目的, 不能与教学目标、教学内容 相衔接, 仅是单纯的为了创设而创设, 学生在目标导向不 明确的状态下学习, 影响了课堂效率。

教师应用信息技术手段的过程中还呈现出一种“貌合 神离”的状态, 很多教师将技术与模式的简单结合理解为 信息化教学模式。由于教师对于各种信息化教学模式的内 涵、规律没有理解, 即使将技术应用到自主学习、协作学 习以及个性化学习等教学模式中, 技术优势也得不到有效 发挥。在电子书包环境下的个性化学习的案例中, 由于教 师对个性化学习本质没有正确的理解, 错把自主学习看做 个性化学习, 因此技术环境下电子书包支持个性化学习的 功能难以发挥作用, 影响了课堂效果, 也不利于学生自主 性的发挥, 影响其个性化发展。

\section{4. 信息化环境下的互动反馈贫乏}

新课改要求发展学生的自主合作探究能力, 因此互动 与反馈在教学过程中显得尤为重要。而信息化教学模式下 技术环境及工具如网络学习空间、评价系统等, 目的都是 为师生、生生之间的互动与反馈提供有利条件, 保证互动 反馈的良好进行。

学生借助技术工具进行互动交流的频率很少。例如在 协作过程中, 学生借助聊天室等交互方式进行交流与协作 的行为几乎不存在, 这也导致学习共同体还是以传统的交 流进行互动, 使得各个成员间的分工不平均, 任务的解答 与展示基本集中在组长身上, 组内其他学生的参与度不高。 又如在借助网络学习空间进行学习时, 只有极少数的学生 通过在线交流的形式进行反馈, 可见学生的主动性不强。

信息化教学模式下要求变革传统的课堂评价形式, 积 极发挥学生的主动性, 使评价更加人性化, 使之体现学生 的全面性发展。但从案例中笔者发现很多课堂缺少多维度、 交互性的总结与评价, 有的甚至忽略评价环节。例如在数 字化课堂环境下的协作学习课堂中, 教师只意识到自己要 综合的评价学生的学习情况, 却忽视了生生之间的相互评 价。又如在基于网络案例的主体性课堂教学中, 教师直接 忽略评价活动, 使得教学过程不完整。

\section{4. 信息技术在课堂教学中的应对策略}

针对信息技术在课堂教学中的应用问题, 结合信息技 术改变课堂教学的作用, 信息技术在课堂教学中的应用应 侧重于信息技术与课堂的有机融合, 为学生的认知、思维 与技能等各方面的发展提供帮助。 


\section{1. 建立教育信息化生态理念, 实现技术与课堂的有效 融合}

生态学将生态环境作为一个整体系统来研究生物与 环境之间的相互作用。信息化教学进程中, 学校作为教育 生态系统, 受到来自信息技术的冲击。为了将信息技术融 入教育生态系统, 有必要在一个综合的、相互联系的生态 环境中看待信息技术与课堂教学的融合。它要求不能割裂 任何原有教育生态系统中的组成部分, 而是应该用系统的、 整体的理念来指导建构和谐共生的教育信息生态系统, 将 新的教学环境, 教学方法和教学理念应用到教学实践中, 实现技术与教学的有效结合。

信息技术与课堂教学相融合的目的是促进人的全面 发展。这一目标符合教育生态系统的核心价值观, 即促进 信息生态学中师生的全面发展, 强调人与技术和教学实践 的和谐互动[17]。因此, 树立生态的信息化教学观, 为信 息技术的有效应用提供良好的生存系统, 是实现技术与课 堂教学融合的助推器。

教育信息化生态观从整体优化的角度考察信息技术 在教育教学中的角色定位与价值, 从以往单一的、单向的 思维转变为多样的、动态的思维, 从关注单一的取向标准 转变为系统的把握整体运行质量及效果, 从关注教师与学 生之间教与学的行为转变为对教师与学生的实际教育学 习需求。教学信息化生态观关注的不是如何破坏原有生态 系统，而是关注既定条件下如何实现信息技术在教育系统 中与师生、教学实践发生适应性的互通与成长, 从而达到 一种平衡状态 [18]。信息技术与课堂教学的深度融合强调 学生是主体, 技术只是辅助, 在学科教学中, 无论信息技 术有多少优势, 都要保证课堂不能喧宾夺主, 要让信息技 术发挥恰到好处的作用, 不要使人与技术的位置发生倒置。 可以看出, 建立良好的教育信息生态观对信息技术的合理 运用具有重要导向作用。

\section{2. 组织有效的技术培训, 提高教师的技术应用能力}

教师和学生是信息技术的实施者, 也是确保信息技术 有效实施的关键, 因此人力资源建设是信息化教学发展的 重要保证。在教育信息化十年发展规划中, 明确指出有必 要加强团队建设, 提高信息技术的应用和服务能力。人力 资源建设主要包括专业技术人员队伍建设、教师建设与学 生建设三方面, 具体实施为:

专业技术人员是教师使用技术的保证。在日常教学生 活中, 不可避免地会出现一些教师无法解决的问题, 如果 不能及时解决, 教师可能会放弃使用信息技术。此外, 教 师信息技术素养的提高需要专业技术人员的帮助。因此, 在学校中配备全职的技术保障人员是必不可少的, 这就需 要上级与学校管理人员积极协调与安排。要继续培养各类 技术人才, 确保学校的技术水平和良好的信息技术氛围, 从而推动信息技术在教育教学中的应用。

教师面对信息技术在观念上虽然发生了质的变化, 但 是在应用上的能力还是欠缺, 很多技术优势没有发挥出来。 而且信息技术日新月异, 教师要想跟得上信息技术的发展 步伐, 就要加强技术应用的水平, 在职培训已成为提高教 师应用技术能力的重要途径。学校应该建立考核与认证一
体化的技术标准, 设置一些技术课程整合的竞赛、培训, 如国家信息技术培训活动等。学校开始可以强制教师参加, 在教师的参与积极性变高后变为非强制性的鼓励政策, 在 教师培训合格后颁发合格证书, 并在之后的教师考核以及 职称评定工作中将信息技术培训成绩纳入其中。通过各种 激励措施, 教师可以提高应用信息技术应用能力, 更新教 育观念, 改进教学方法, 提高教学质量[19]。

学生是课堂教学的主体, 也是信息技术应用的主要对 象, 因此提高学生的信息技术素养也非常重要。只有当教 师和学生的信息技术能力共同得到提高才能更好地促进 信息化课堂的有效展开。为了提高学生的信息技术能力, 鼓励学生主要通过培养兴趣来体验信息化课堂的乐趣, 从 而引导他们积极利用信息技术在教学中的能力, 促进他们 的学习。

\section{3. 打破时间与空间限制, 拓展交流渠道}

课堂教学中信息技术在呈现知识、情境创设上具有优 势, 能够利用信息技术工具的多样性为学生创设良好的问 题情境, 为学生呈现学习内容。除此之外, 还被学生用作 认知工具和资源环境, 根据教师的任务活动进行探究学习。 通过这些教学活动可以看到, 技术正在改变课堂, 不断促 进教与学方式的变革。例如在网络案例学习中网络学习空 间为学生、教师、家长同时开放了交流平台, 数字化课堂 环境下也为协作学习提供数字化交流平台。但是在看到优 势的同时不能忽视弊端, 通过分析发现, 信息技术的应用 过程主要以基本功能的使用为主, 而在学生开展认知与探 究过程中技术支持功能缺乏。

任何形式的学习都离不开沟通,沟通是确保课堂有效 性的重要手段。当前中国已经初步形成了覆盖全国的资源 服务体系, $87.1 \%$ 的学校已经实现了网络接入 [20]。网络 环境的优势已经足以支撑现代化信息技术的使用, 完全可 以打破教室学习的局面, 实现不同地点、不同时间、不同 对象的多边互动。因此, 要充分发挥信息环境的优势, 真 正实现无处不在的学习, 促进学生的多元化学习。

师生之间、生生之间借助信息化环境互动贫乏很大程 度上是由于教师、学生没有看到网络交流对教学的影响力。 因此, 应该培养交流对象借助信息技术进行交流的意识, 认识到网络交流可以很好地作用于学习, 有利于提高学习 效率，增进同伴间的感情。

面对教师，学校应该鼓励和督促教师使用网络传播。 例如, 可以组织教师进行汇报交流, 反馈各学科应用信息 技术的情况; 又如, 转变备课形式, 组织教师借助网络学 习空间进行集体备课, 推动着教师必须应用平台进沟通、 交流并完成集备。通过这种激励方式, 教师可以体验到网 络传播的优势和意义。面对学生, 教师应该采取鼓励与强 制方式让学生体验到利用网络交流进行学习的意义。教师 可以通过开展多互动的教学, 例如基于资源的主题教学、 基于探究的学习等, 学生不得不借助平台或其他软件进行 资源、经验的分享与交流; 又如, 教师可以创建“聊天室” 或“论坛”, 每周进行一次课程讨论, 让学生畅所欲言, 互 相学习进步。 


\section{4. 有效运用评价方式, 实现多元化评价}

信息技术的有效运用离不开有效的教学反馈, 教学反 馈的有效性离不开有效的教学评价, 教学评价的有效性离 不开多元化的评价标准。信息化课堂更加关注教学活动是 否满足教与学的需要, 目的是促进人的全面发展, 因此教 学过程是处于动态的、不断变化的。由此在评价过程中不 能像传统的教学评价一样只关注某个方面, 不能以偏概全, 要全面的考虑教学过程的各个环节, 不能过分关注结果, 更要考虑到学生的发展需要。因此, 检验信息技术是否有 效利用取决于是否坚持了开放的、动态的多元化的评价准 则[21]。

信息化环境下的课堂教学往往不能很好地利用多元 评价的优势, 只有极少数教师组织学生借助反馈系统进行 交互性评价, 更多的仍然是基于成绩定好坏, 而不是考虑 到学生发展性的需求。因此, 通过信息化环境的过程评价 功能的支持, 可以充分发挥教师与学生的能动性, 实现评 价主体多元化, 评价方法多样化, 促进学生全面发展。

在课堂教学过程中, 应充分利用信息评价工具的作用。 教师应该很好的借助各种评价数据反馈系统, 更多的目光 聚焦到每个学生的活动过程, 看到每个学生的发展特点, 不再拘泥于终结性评价。教师与学生的多样化评价主体的 参与, 保证了评价的多元化, 有利于学生的全面发展。

在最终学习成果中将总结性评价与过程性评价相结 合, 在最终的总结性评价中应该体现过程性评价。当前大 多还以总结性评价为主, 总结性评价以其概括性高、测验 范围覆盖广的特点一直作为判定最终学习结果的方式, 但 这一方式正在随着信息技术的渗透逐渐弱化, 过程性评价 的作用开始显现出来。因此, 学校应该从规范上明确过程 性评价的作用, 从意识上引导教师从不同角度、不同方面 评价学生, 从而保证更有效的评价学生。此外, 学校还应 该从制度上明确形成性评价结果的应用, 学校应该将平时 的评价结果进行量化管理, 并按照比例算入学生的最终学 习结果中, 将总结性评价与过程性评价结果相结合, 使成 绩更能反映学生的全面性, 从而更有效地评价学生。

\section{5. 结论}

当今现代化课堂教学已离不开信息技术的普及与应 用, 但信息技术与课堂教学深度融合过程中还存在着一系 列问题, 本文针对信息技术在教学中的定位不明确、教师 的意识与能力有欠缺、课堂应用不恰当、互动反馈贫乏等 问题进行了分析讨论, 并给出了 4 点对策建议, 即信息技 术的课堂教学应用应从宏观上树立良好的教育信息化生 态观，实现信息技术与课堂教学的有效融合; 通过组织有 效的技术培训, 提高教师的技术应用能力, 实现技术应用 能力有效发挥; 通过全面落实师生间的多维互动, 打破时 间与空间限制, 拓展交流渠道; 通过充分应用多元化评价 准则, 实现评价的多元化, 从而达到优化课堂教学、提高 教学质量、更利于信息时代人才的培养的目的。

\section{致谢}

本文为北京市社会科学基金” 典型复杂工程问题在信 息类专业创新性实践教学环节中的应用研究”(16JYC016); 教育部人文社会科学研究青年基金” 基于复杂工程问题的 迁移学习在创新性实践教学中的应用研究” (17YJC880137) 的阶段性成果之一。

\section{参考文献}

[1] 董德森.信息技术在高等教育中的应用价值[J].黑龙江高教 研究, 2013, 31 (2): 34-36。

[2] 中华人民共和国教育部.基础教育课程改革纲要（试行） [EB/OL]. [2001-06-08]。

[3] 中华人民共和国教育部.国家中长期教育改革和发展规划 纲要 (2010-2020) [N].人民日报, 2010-7-30。

[4] 中华人民共和国教育部.教育信息化十年发展规划 （2011-2020年）[EB/OL]. [2012-03-13]。

[5] 徐继存, 车丽娜. 教学的技术嵌入及其规约 [J]. 课程. 教 材·教法, 2015 (7): 12-17.

[6] 余胜泉, 陈玲.1:1课堂网络教学环境下的教学变革[J].中国 电化教育, 2007 (11): 25-29。

[7] 王宇飞.中学信息技术课程专题学习网站的设计[J].现代教 育技术， 2007 (12): 90-92。

[8] 祝智庭, 管玉琪. 教育变革中的技术力量[J].中国电化教育, 2014 (1): 1-9。

[9] 徐松.Pad上课一一场即将到来的课堂教学变革— i Pad引 发课堂教学的“三个变化”[J].当代教育科学, 2013 (4): 29-31。

[10] 户锦耀.论信息技术应用所引发的课堂教学变革 [J]. 现代 教育论丛，2001 (5): 61-64。

[11] 钟志贤.信息化教学模式——理论建构与实践[M].北京: 教 育科学出版社, 2005: 55-60。

[12] 何克抗, 吴娟.信息技术与课程整合的教学模式研究之一 ——教学模式的内涵及分类[J].现代教育技术, 2008 (7): 7-8。

[13] 梁文鍂. 大数据时代——课堂教学将迎来真正的变革 [J]. 北京教育学院学报 (自然科学版), 2013 (8): 14-16。

[14] 郭玉莲.课堂教学模式改革探论 [J]. 教育理论与实践, 2012 (10): 57-60。

[15] 车丽娜等. 山东省基础教育课堂教学改革的分析与反思 [J]．教育研究， 2015 (12): 146-153。

[16] Emmanuel G M. Technological changes: Its impact on Man and Society [M]. New York: New American Library, 1970. 60。

[17] 余胜泉.教育信息化的生态观[J].教育技术学报, 2007 (11): 24-29.M。 
[18] 余胜泉, 赵兴龙.基于信息生态观的区域教学信息化推进 [J]．中国电化教育， 2009 (8): 33-40。

[19] 王吉庆. 信息技术课程与教学论 [M]. 浙江: 浙江教育出版 社, 2003。
[20] 中华人民共和国教育部.2015年12月教育信息化工作月报 [EB/OL]. [2016-01-28]。

[21] 刘斌.信息化教学有效性的理论思考--对信息化教学本质的 再认识 [J]. 现代教育技术, 2013, 23 (3): 26-30。 